\title{
ANÁLISE DO COMPORTAMENTO DOS PREÇOS DO BOI GORDO E DO BOI MAGRO NA PECUÁRIA dE CORTE PAULISTA, NO PERÍODO DE 1995 A 2006 ${ }^{1}$
}

\author{
Raquel Castellucci Caruso Sachs ${ }^{2}$ \\ Eder Pinatti ${ }^{3}$
}

Resumo - Este trabalho analisou os efeitos dos choques nos preços do boi gordo sobre o comportamento dos preços do boi magro no estado de São Paulo, no período de janeiro de 1995 a janeiro de 2007. Utilizaram-se na análise os testes de raiz unitária de Dickey-Fuller Aumentado (ADF), de causalidade de Granger, de co-integração de Johansen, o método de auto-regressão vetorial (VAR), decomposição dos erros da variância e função de resposta de impulso. Os resultados mostraram que existe relação unidirecional entre os preços das duas categorias animais, o que confirma a importância do preço do boi gordo na formação do preço do boi magro, embora os preços não apresentem relação de equilíbrio no longo prazo.

Palavras-Chave: Boi gordo, boi magro, co-integração, modelo de auto-regressão vetorial (VAR), pecuária de corte.

Recebido em: 10/07/2007 Aceito em: 18/09/2007

Pesquisadora científica do Instituto de Economia Agrícola.

E-mail: raquelsachs@iea.sp.gov.br

3 Pesquisador científico do Instituto de Economia Agrícola. E-mail: pinatti@iea.sp.gov.br 


\section{Introdução}

A bovinocultura de corte é uma das principais atividades agrícolas do estado de São Paulo, ficando atrás apenas da cana-de-açúcar. Em 2005, o volume paulista de carne bovina produzida foi de 78,21 milhões de arrobas (@), sendo o respectivo valor da produção agropecuária (VPA) de $\mathrm{R} \$ 4,3$ bilhões, que corresponde 15,4\% do VPA total do Estado, que foi de 27,82 bilhões, segundo dados do Instituto de Economia Agrícola (IEA).

As exportações da carne bovina somaram US $\$ 3,16$ bilhões em 2005, o que representou 2,7\% da exportação nacional. De 2004 para 2005, houve variação positiva de $22,1 \%$, que somente não foi maior em virtude do foco de febre aftosa, em outubro de 2005, no estado do Mato Grosso do Sul. Nos seis primeiros meses de 2006, já foram exportados US\$1,72 bilhões, uma evolução de 16,0\% em relação ao mesmo período de 2005 , quando ainda não se havia detectado o foco de aftosa.

Nas últimas décadas, vem ocorrendo progresso tecnológico na cadeia produtiva, especialmente na fase de produção animal, e essas mudanças tecnológicas refletem no volume produzido e na oferta de carne e, conseqüentemente, nos preços dos animais das diversas categorias.

Diante da grande representatividade da pecuária no contexto econômico estadual, da recente evolução da pecuária bovina e das mudanças na economia brasileira, a análise do comportamento dos preços (em especial, do boi gordo e do boi magro) dentro da cadeia produtiva é de extrema importância, já que é indispensável ao correto planejamento da atividade.

\section{Objetivos}

O objetivo deste trabalho é analisar a relação entre os preços do boi gordo e do boi magro no estado de São Paulo, no período de janeiro de 1995 a janeiro de 2007. Especificamente, pretende-se verificar como 
variações no preço do boi gordo se transmitem para o preço do boi magro, ao longo do tempo.

\section{Pecuária de corte e mercado do boi gordo}

A produção de bovinos de corte envolve fases distintas, que são cria, recria e engorda. A fase de cria compreende a reprodução e o crescimento dos bezerros até a desmama, e o bezerro desmamado é o produto final desta fase. Na recria, que se inicia com o desmame e termina com os animais indo para a fase de engorda ou para a fase de cria como matrizes ou reprodutores, os produtores adquirem e recriam os bezerros desmamados e produzem bois magros e, ou, vacas para cria. A fase de engorda consiste na etapa final, em que bois magros (principal insumo da engorda) ou vacas magras são engordados até atingirem o peso de abate; o produto final desta fase e também da pecuária de corte são o boi gordo (principal produto) e a vaca gorda, que são destinados ao abate, sendo vendidos para os abatedouros ou frigoríficos que abastecem o mercado consumidor interno e externo.

Os criadores também podem atuar em duas fases e há, inclusive, os que atuam nas três fases, o chamado ciclo completo. Assim, fazem da produção do bezerro até a produção do boi gordo, passando pelo garrote e boi magro.

Para cada uma dessas categorias de animal há um mercado, onde esses animais são comercializados entre criadores que desenvolvem as fases complementares da produção e onde se definem os seus preços de comercialização.

Os preços dos animais vivos apresentam acentuada sazonalidade dentro do ano, relacionada com a disponibilidade de animais gordos em ponto de abate, que, por sua vez, é decorrente da maior ou da menor disponibilidade de pastagens ao longo das estações do ano. Os preços dos animais vivos apresentam também ciclos plurianuais de acréscimo e decréscimo, 
conhecidos como ciclo pecuário, "no qual a queda na cotação do boi gordo deflagra retração nas cotações das categorias intermediárias até que as matrizes passam a serem descartadas, acentuando o excesso de oferta de carne bovina e a queda dos preços. Os preços só tendem a reagir quando se verifica redução na disponibilidade de boi gordo, o que, dado o sistema de exploração extensivo empregado, deverá ocorrer três a quatro anos após o início do descarte de matrizes" (Toledo e Santiago, 1984).

A existência desses ciclos na pecuária de corte deve ser levada em consideração quando se definem políticas de financiamento para o setor, pois, adotadas em momento inadequado, podem vir a reforçar fases de preços altos, que prejudicam os consumidores de carne, ou de preços baixos dos animais, que prejudicam os produtores. O ideal, em matéria de políticas para a pecuária, são as medidas que encurtam as fases ascendente ou descendente do ciclo pecuário ou estabilizam o preço da carne em patamar mediano.

Em trabalhos encontrados na literatura, estudou-se a transmissão de preços entre diferentes níveis de mercado, como o trabalho de Bliska (1989), que analisou a transmissão de preços de carne bovina entre produtor, atacado e varejo. Outros trabalhos desenvolveram modelos de previsão de preços na pecuária, dentre os quais se destacam os de Kassouf (1988) e Bacchi (1994), ou, ainda, transmissão de preços entre diferentes segmentos da pecuária de corte, dentre eles, os de Hasegawa (1995) e Bacchi (1999).

Hasegawa (1995), ao trabalhar com séries de preços referentes ao período de 1970 a 1994, concluiu que "o preço do boi gordo é o único a influenciar sistematicamente os demais preços da pecuária de corte" e que "na fase ascendente do ciclo, o mercado do boi gordo domina os demais mercados como fonte da variação dos preços, enquanto que, na fase descendente, as outras categorias passam a influenciar muito mais os outros mercados quanto à variação de preços". 
Bacchi (1999), ao analisar a transmissão de preços entre boi gordo, bezerro e boi magro, no período de janeiro de 1981 a fevereiro de 1995, concluiu que existe alto grau de integração entre os setores de produção desses segmentos da pecuária e que as variações de preços do boi gordo são transmitidas, rapidamente, para os outros dois segmentos da cadeia produtiva.

$\mathrm{O}$ fato de o mercado de boi gordo ser o mais bem coberto por informações de preço torna natural, que nos negócios com outras categorias, a cotação do preço do boi gordo seja tomada como referência.

Os pecuaristas formam um conjunto muito heterogêneo, no que se refere à utilização de tecnologia e de escala de produção. Nas últimas décadas, vem ocorrendo progresso tecnológico na cadeia produtiva, especialmente na fase de produção animal, com melhoria das pastagens, maior utilização de suplementação mineral e de forrageiras de inverno, melhorias no manejo dos animais e também no melhoramento genético do rebanho, setores nos quais estão ocorrendo investimentos, seja por parte dos criadores de gado de elite, seja por parte dos criadores comerciais. Essas mudanças tecnológicas, apesar de ainda pequenas, refletem no volume produzido e na oferta de carne, na sazonalidade da produção e também nos preços dos animais de diversas categorias e dos produtos finais, alterando, inclusive, a repartição dos ganhos econômicos entre os agentes da cadeia produtiva.

O cenário econômico nacional sofreu profundas transformações nas últimas décadas, com destaque para o plano de estabilização econômica brasileira (Plano Real) em 1994, cujas conseqüências foram maior estabilidade econômica do país e consolidação do novo perfil administrativo. Os conceitos da cadeia produtiva estão sendo revistos, já que se enfrenta uma nova realidade, com margens de lucros mais deprimidas para os produtores de bovinos de corte, em que se deve prevalecer o planejamento da atividade, tanto na parte de custo como na receita, para que o produtor possa tornar a equação positiva. 
A hipótese deste trabalho, baseada no conhecimento empírico dos pecuaristas, é que as variações no preço do boi gordo continuem influenciando a formação de preço das outras categorias de bovinos, apesar da evolução recente da pecuária bovina (caracterizada pelo encurtamento do ciclo de produção dos animais, pela redução da sazonalidade da oferta de carne, pelo aumento da taxa de desfrute dos rebanhos) e das mudanças na economia nacional, que ocasionaram margens de lucro mais apertadas.

Assim, torna-se imprescindível a análise do comportamento dos preços da bovinocultura de corte, em especial, do boi gordo e do boi magro.

\section{Dados e métodos}

\subsection{Dados}

Foram utilizadas duas séries de tempo com observações mensais relativas às seguintes variáveis: cotação de preço nominal do boi gordo no estado de São Paulo, em R\$/@, e cotação de preço nominal do boi magro no estado de São Paulo, em R $\$$ /unidade, ambos no período de janeiro de 1995 a janeiro de 2007. Os dados foram obtidos dos levantamentos realizados pelo Instituto de Economia Agrícola (IEA) e publicados no Anuário de Informações Estatísticas da Agricultura (1995-2005) e da revista Informações Econômicas (2007).

Os dados do preço do boi gordo e do boi magro foram utilizados na sua forma nominal, ao invés da real, devido à metodologia empregada neste trabalho, pois o uso de algum tipo de deflator pode contaminar as séries, ao inserir elementos estranhos à série original, e, conseqüentemente, levar à identificação de modelos deturpados. Portanto, questões relativas à deflação de preços e a qual deflator pode ser utilizado devem ser analisadas cuidadosamente, pois esse procedimento, possivelmente, interferiria nos resultados da análise. Pino e Rocha (1994) demonstraram a possibilidade de se trabalhar, perfeitamente, com séries de tempo 
nominais, em que as séries deflacionadas são aproximadamente equivalentes ao modelo com série sem deflação, ainda mais em período em que o nível de inflação é baixa, como é o caso deste estudo.

Nesse estudo, as variáveis foram utilizadas na forma de logaritmo; então, os valores dos próprios coeficientes fornecem diretamente as respectivas elasticidades. Logo, as variáveis logarítmicas dos preços do boi gordo e do boi magro foram denominadas $\mathrm{Lbg}$ e $\mathrm{Lbm}$, respectivamente.

\subsection{Métodos}

Trabalhos empíricos baseados em dados de séries temporais, geralmente, admitem que essas séries são estacionárias. Em uma regressão pode-se obter um alto valor do coeficiente de determinação $\left(\mathrm{R}^{2}\right)$, embora não exista relação significativa entre as variáveis. Essa situação é denominada de regressão espúria (Gujarati, 1995).

Segundo Harris (1995), uma série estacionária tende a retornar ao seu valor médio e tem variância finita, enquanto uma série não-estacionária tem média diferente para cada ponto no tempo e sua variância aumenta com o tamanho da amostra.

Para determinar a ordem de integração das variáveis foi utilizado o teste de raiz unitária Dickey-Fuller Aumentado (ADF), conforme apresentado em Dickey e Fuller (1979 e 1981). Os valores críticos para os testes de raiz unitária individuais foram obtidos de Mackinnon (1991), enquanto os valores críticos para os testes conjuntos foram extraídos de Dickey e Fuller (1981).

O teste de raiz unitária ADF utiliza a seguinte auto-regressão:

$$
\Delta y_{t}=\alpha+\beta t+\left(\rho_{1}-1\right) y_{t-1}-\sum_{j=1}^{p-1} \rho_{j+1} \Delta y_{t-j}+e_{t},
$$


em que $\alpha$ é o intercepto; $\mathrm{t}$, tendência; $\Delta$, operador diferença $\left(\Delta \mathrm{Y}_{\mathrm{t}}=\mathrm{Y}_{\mathrm{t}}-\right.$ $\left.\mathrm{Y}_{\mathrm{t}-1}\right)$, e $\Delta \mathrm{Y}_{\mathrm{t}-\mathrm{j}}$, própria variável dependente, diferenciada e defasada. $\mathrm{O}$ número de defasagens é determinado pelo menor valor do Critério de Schwarz e visa eliminar a autocorrelação dos resíduos, e e representa a estrutura de erro, que é identicamente e independentemente distribuída.

O teste de raiz unitária testa a hipótese nula de presença de raiz unitária $(\rho=1)$ contra a hipótese alternativa de que a série é estacionária $(\rho<1)$, considerando a presença, ou não, da constante e, ou, da tendência, denominados testes $\tau$ (modelo sem constante e sem tendência), $\tau$ (modelo com constante e sem tendência) e $\tau$ (modelo com constante e tendência), e também possibilita a realização de testes conjuntos sobre o parâmetro de raiz unitária e a presença, ou não, do intercepto e, ou, tendência, denominados testes $\phi$ (testa a hipótese nula de presença de raiz unitária e de que não há intercepto) e $\phi$ (testa a hipótese nula de presença de raiz unitária, em que há interceptổo, mas não há tendência).

Para verificar o relacionamento entre variáveis, pode-se realizar o teste de causalidade de Granger, de acordo com Granger (1969). Esse conceito de causalidade diz que a "variável $A$ causará a variável $B$ no sentido de Granger", quando os valores passados de $A$ ajudarem a prever, de maneira mais precisa, o comportamento futuro de $B$, ao invés de se utilizarem somente valores passados de $B$. Esse teste deve ser conduzido com as variáveis estacionárias.

O teste de co-integração procura identificar se duas ou mais variáveis integradas, de mesma ordem, possuem relação de equilíbrio no longo prazo. A interpretação econômica de co-integração, segundo Harris (1995), é de que há relação de equilíbrio de longo prazo entre as séries; então, mesmo que elas possam conter tendências estocásticas, ou seja, não serem estacionárias, apresentam tendência conjunta de longo prazo. A diferença entre as séries será estável, ou seja, estacionária. Portanto, o conceito de co-integração mimetiza a existência de um equilíbrio de longo prazo, para o qual um sistema econômico converge ao longo do tempo. 
No teste de co-integração utilizou-se a abordagem de Johansen, segundo Johansen e Juselius (1990), procedimento que se baseia na seguinte versão reparametrizada de um modelo VAR (p).

$$
\Delta y_{t}=\Gamma_{1} \Delta y_{t-1}+\cdots+\Gamma_{p-1} \Delta y_{t-p+1}+\Pi y_{t-1}+\mu+\varphi d_{t}+e_{t},
$$

em que y é um vetor com $k$ variáveis; e $\sim \mathrm{N}(0, \Sigma)$ e $\mathrm{E}\left(\mathrm{e},(\mathrm{e})^{\mathrm{t}}\right)=0$, para qualquer t diferente de s; e d, um vetor de variáveis binárias para captar a variação estacional e, ou, variáveis de intervenção.

A metodologia de co-integração, de Johansen, apresenta três situações distintas. Se o posto de $\pi$ for completo (posto igual a $r$ ), isto é, se houver $\mathrm{r}=\mathrm{k}$ colunas linearmente independentes, então y será estacionário; se o posto de $\pi$ for zero $(\mathrm{r}=0)$, então $\Delta \mathrm{y}$ será estấcionário e não haverá relacionamento de co-integração; finalmente, se $0<\mathrm{r}<\mathrm{k}$ e se existirem matrizes $\alpha$ e $\beta$ de dimensão $\mathrm{k} \times \mathrm{r}$, tais que $\pi=\alpha \beta^{\mathrm{t}}$ e o vetor $\beta^{\mathrm{t}} \mathrm{y}$ for estacionário, haverá, portanto, $r$ vetores de co-integração (as $r$ colùnas de $\beta$ ).

Neste estudo, para testar a presença de vetores de co-integração, foi utilizada a estatística $\lambda$. Para testar a hipótese nula de que existem

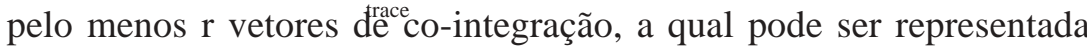
matematicamente da forma abaixo, conforme Johansen e Juselius (1990), citados por Margarido (2002):

$\mathrm{H}_{0}: \lambda_{\mathrm{i}}=0 \quad \mathrm{i}=\mathrm{r}+1, \ldots, \mathrm{n}$,

ou seja, somente os primeiros $r$ autovalores $(\lambda)$ são diferentes de zero. Essa restrição pode ser imposta a diferentes valores de r. O próximo passo consiste na comparação do valor do logaritmo da função de verossimilhança do modelo com a restrição relativa ao logaritmo da função de verossimilhança do modelo sem restrição. Esse teste é denominado estatística $\lambda_{\text {trace }}$ e é representado, em termos algébricos, por 
$\lambda_{\text {trace }}=-2 \log (Q)=-T \sum_{i=r+1}^{n} \log (1-\hat{\lambda}) \quad r=0,1,2, \ldots, n-2, n-1$,

em que $\mathrm{Q}=($ função de verossimilhança restrita maximizada $\div$ função de verossimilhança sem restrição maximizada).

Os efeitos dinâmicos de choques do preço do boi gordo sobre o preço do boi magro foram estudados pela análise de Auto-Regressão Vetorial (VAR), que é uma metodologia indicada para estimar modelos que envolvem inter-relações complexas das variáveis. Um modelo VAR pode ser representado por

$$
y_{t}=\phi_{1} y_{t-1}+\phi_{2} y_{t-2}+\ldots+\phi_{t} y_{t-p}+e_{t},
$$

em que y é um vetor ( $\mathrm{n} \times 1$ ) com variáveis de interesse; $\phi$, matriz de coeficientes de ordem $\mathrm{k} \mathrm{x} \mathrm{k}$; e e, vetor coluna de erros que contém um processo ruído branco (white noise), com as seguintes propriedades:

$\mathrm{E}\left(e_{t}\right)=0$ para todo t $e$,

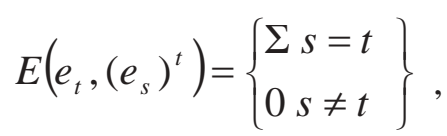

em que $\Sigma$ é a matriz de co-variância, a qual pressupõe ser definida como positiva, enquanto os termos são serialmente não-correlacionados, mas podem ser contemporaneamente correlacionados.

A equação (4) pode ser escrita por $\phi(B) y_{t}=e_{t}$,

em que $\phi$ (B) é um polinômio com operador de defasagem, tal que $B^{j} y_{t}=y_{t-j}$, para $\mathrm{j}$ inteiro, e a matriz $\phi_{0}=\mathrm{I}$. 
Se o processo for estacionário, a equação (5) poderá ser representada na forma de média móvel:

$y_{t}=\Theta(B) e_{t}$,

em que $\Theta(B)$ é estimado conhecendo-se $\phi(B)$, sendo um polinômio matricial de ordem infinita.

Segundo Margarido (2002), ao utilizar modelos VAR ou VEC (modelo de auto-regressão vetorial com correção de erro), um dos principais objetivos é examinar os efeitos de choques individuais sobre a dinâmica do sistema, sendo necessário, para isso, efetuar alguns ajustes em relação à matriz de variância-covariância dos resíduos $(\Sigma)$, pois, geralmente, essa não é uma matriz diagonal, e os elementos de e $\mathrm{t}_{\mathrm{t}}$ podem ser correlacionados, de tal forma que um choque em $e_{i, t-j}$ poderá ser acompanhado por alterações contemporâneas nos demais. Dessa maneira, é necessário diagonalizar a matriz de variância-covariância para evitar que choques sobre determinada variável contaminem todo o sistema, impedindo a análise de efeito individual desse choque sobre o comportamento da variável de interesse.

Com esse procedimento, é possível obter, para cada variável, a decomposição da variância dos erros de previsão $k$ períodos à frente, em percentagem, o que permite aferir o poder explicativo de cada um dos componentes do modelo sobre os demais e, ainda, obter os comportamentos das variáveis em resposta a choques individuais em quaisquer componentes do sistema.

Supondo-se, agora, que cada série do vetor $\mathrm{y}_{t}$ seja integrada de ordem $\mathrm{d}$ - I(d), isto é, seja não-estacionária devido à presença de raiz unitária (tendência estocástica), então um VAR, nas diferenças, deve ser estimado. O VAR, nas diferenças, pode ser representado por:

$\phi^{*}(B)(1-B) y_{t}=e_{t}$, 
em que $\phi^{*}(L)=I+\phi_{1}^{*} L+\ldots+\phi_{p-1}^{*} L^{p-1}$ e $\phi_{j}^{*}=-\sum_{j=i+1}^{p} \phi_{j}$

\section{Resultados e discussão}

Apresentam-se, inicialmente, os resultados dos testes de raiz unitária, seguidos dos testes de causalidade, co-integração e, por fim, os do modelo de Auto-regressão Vetorial (VAR).

Antes de realizar o teste de raiz unitária, foi necessário determinar o número de defasagens a serem utilizadas em cada teste para eliminar uma possível autocorrelação dos resíduos. Pelo critério de Schwarz (BIC), foram necessárias duas defasagens para a variável preço do boi gordo e para a variável preço do boi magro, ambas em nível, conforme mostra a Tabela 1.

Tabela 1 - Determinação do número de defasagens segundo o Critério de Informação de Schwarz (BIC), para executar o teste de raiz unitária do tipo ADF para as variáveis $L b g$ e $L b m$, janeiro de 1995 a janeiro de 2007

\begin{tabular}{lcc}
\hline Variável & $\begin{array}{c}\text { Valor Mínimo do Critério de } \\
\text { Informação (BIC) }\end{array}$ & $\begin{array}{c}\text { Defasagem(ns) efetivamente } \\
\text { utilizada(s) no modelo ARMA }\end{array}$ \\
\hline \hline$L b g^{1}$ & $\operatorname{BIC}(2,0)$ & Duas defasagens \\
$\triangle \mathrm{Lbg}^{2}$ & $\mathrm{BIC}(3,0)$ & Três defasagens \\
$L b m^{1}$ & $\mathrm{BIC}(2,0)$ & Duas defasagens \\
$\triangle L b m^{2}$ & $\mathrm{BIC}(2,0)$ & Duas defasagens \\
\hline
\end{tabular}

${ }^{1}$ Variável em nível.

${ }^{2}$ Variável diferenciada.

Fonte: Dados Básicos do Instituto de Economia Agrícola (1995-2007).

O Critério de Informação de Schwarz (BIC) indicou a necessidade de incluir três defasagens na variável preço do boi gordo diferenciada e duas defasagens na variável preço do boi magro diferenciada. 
Os resultados do teste de raiz unitária ADF estão apresentados na Tabela 2. Pode-se verificar, pelas significâncias dos testes $\tau$ e $\phi$, que as variáveis em nível não são estacionárias, portanto, não se rejeita a hipótese inicial de que há raiz unitária. Os testes conduzidos com as variáveis diferenciadas mostraram que elas são estacionárias, considerando todos os três níveis de significância adotados para cada estatística implementada. Portanto, as duas variáveis foram consideradas diferenças estacionárias, ou seja, integradas de ordem um (I(1)), pois foi necessária a aplicação de uma diferença de ordem um para tornar cada uma estacionária.

Tabela 2 - Resultados do teste de raiz unitária Dickey-Fuller Aumentado (ADF) para as variáveis $L b g$ e $L b m$, janeiro de 1995 a janeiro de $2007^{1}$.

\begin{tabular}{lrrrrr}
\hline Variável & \multicolumn{1}{c}{$\tau_{\tau}{ }^{2}$} & \multicolumn{1}{c}{$\phi_{3}{ }^{2}$} & \multicolumn{1}{c}{$\tau_{\mu}{ }^{2}$} & \multicolumn{1}{c}{$\phi_{1}{ }^{2}$} & \multicolumn{1}{c}{$\tau^{2}$} \\
\hline \hline $\mathrm{Lbg}^{3}$ & $-1,87$ & 1,88 & $-1,07$ & 1,67 & 1,37 \\
$\triangle \mathrm{Lbg}^{4}$ & $-7,21$ & 26,35 & $-7,19$ & 25,84 & $-6,81$ \\
$\mathrm{Lbm}^{3}$ & $-1,35$ & 0,95 & $-0,65$ & 2,41 & 2,06 \\
$\triangle L b m^{4}$ & $-6,69$ & 22,54 & $-6,72$ & 22,55 & $-6,26$ \\
\hline
\end{tabular}

${ }^{1}$ Valores críticos para $\tau \tau$ e $\tau$, obtidos conforme Mackinnon (1991), correspondem, respectivamente, a $-3,1^{\tau} 44^{4} 9,-2,5772$ e $-1,6168$, em nível de $10 \% ;-3,4413,-2,8814$ e $-1,9420$, em nível de $5 \% ;-4,0237,-3,4764$ e $-2,5798$, em nível de $1 \%$. Os valores críticos para $\phi$ e $\phi$ foram obtidos em Dickey e Fuller (1981) e correspondem, respectivamentê, a 5,47 e 3,86, em nível de $10 \% ; 6,49$ e 4,71, em nível de 5\%; 8,73 e 6,70 , em nível de $1 \%$.

${ }^{2} \tau$ : modelo sem constante e sem tendência; $\tau$ : modelo com constante e sem tendência; $\tau$ : modelo com constante e tendência; $\phi$ : ẗesta a hipótese nula de presença de raiz unitária e de que não há intercepto; e $\phi^{1}$ :testa a hipótese nula de presença de raiz unitária, de que há intercepto, mas não hả tendência.

${ }^{3}$ Variável em nível.

${ }^{4}$ Variável diferenciada.

Fonte: Dados Básicos do Instituto de Economia Agrícola (1995-2007). 
Antes da aplicação do teste de causalidade foi necessário determinar o número de defasagens a serem utilizadas. Para isso, empregou-se o Critério de Informação de Akaike (AIC), que resultou em um modelo auto-regressivo para a primeira defasagem ${ }^{4}$.

Foi realizado o teste de causalidade de Granger, para verificar o grau de relacionamento entre as variáveis do sistema.

A hipótese nula $\left(\mathrm{H}_{0}\right)$, de que o preço do boi magro "não causa, no sentido de Granger", o preço do boi gordo não pode ser rejeitada, pois a probabilidade de se rejeitar a hipótese nula e de ela ser verdadeira é igual a $31,98 \%$.

Porém, a hipótese nula de que o preço do boi gordo "não causa, no sentido Granger," o preço do boi magro foi rejeitada, pois a probabilidade de se rejeitar a hipótese nula de que o boi gordo não causa o preço do boi magro e de essa hipótese ser verdadeira é igual a $0,35 \%$. Portanto, a possibilidade de se rejeitar algo que é verdadeiro é muito pequena; assim, a hipótese nula pode ser rejeitada e a hipótese alternativa (preço do boi gordo causa o preço do boi magro), aceita. Esse resultado indica que o preço do boi magro é influenciado pelo comportamento do preço do boi gordo, conforme esperado, ou seja, o sentido de causalidade é unidirecional (Tabela 3).

4 Foram utilizados outros tipos de critérios de informação para determinação do número de defasagens (AICC: Critério de Informação de Akaike Corrigido; FPE: Erro de Predição Final; HQC: Critério de Hanna-Quinn e SBC: Critério Bayesiano de Schwarz). O AICC e SBC apresentaram o mesmo resultado do Akaike; portanto, optou-se por esse critério. 
Tabela 3 - Resultados dos testes de causalidade de Granger para as variáveis $L b g$ e $L b m$, janeiro de 1995 a janeiro de 2007

\begin{tabular}{lccc}
\hline \multicolumn{1}{c}{ Teste } & $\begin{array}{c}\text { Qui- } \\
\text { Quadrado }\end{array}$ & $\begin{array}{c}\text { Graus de } \\
\text { Liberdade }\end{array}$ & $\begin{array}{c}\text { Prob>Qui- } \\
\text { Quadrado }\end{array}$ \\
\hline \hline $\begin{array}{l}\text { Preço do boi magro não } \\
\text { causa preço do boi gordo }\end{array}$ & 0,99 & 1 & 0,3198 \\
$\begin{array}{l}\text { Preço do boi gordo não } \\
\text { causa preço do boi magro }\end{array}$ & 8,52 & 1 & 0,0035 \\
\hline
\end{tabular}

Fonte: Dados Básicos do Instituto de Economia Agrícola (1995-2007).

Dado que o teste de raiz unitária mostrou que as duas variáveis, $L b g$ e Lbm, são I(1), realizou-se o teste de co-integração para verificar se essas variáveis tendem a uma situação de equilíbrio no longo prazo. Antes da aplicação do teste de co-integração, foi necessário determinar o número de defasagens a serem utilizadas. Para isso, empregou-se o Critério de Informação de Akaike (AIC), que resultou em um modelo auto-regressivo para a segunda defasagem.

Pelo teste de co-integração, de Johansen, constatou-se que não há vetor de co-integração. A hipótese nula $\left(\mathrm{H}_{0}\right)$, de que não há nenhum vetor de co-integração $($ Rank $=0)$, não foi rejeitada em favor da hipótese de alternativa $\left(\mathrm{H}_{1}\right)$ de que tenha, pelo menos, um vetor de co-integração $($ Rank $>0)$, uma vez que o valor calculado da estatística traço $\left(\lambda_{\text {traço }}\right)$ é inferior ao seu respectivo valor tabelado $(6,81<12,21)$. Os resultados podem ser visualizados na Tabela $4^{5}$.

5 A estatística $\lambda_{\text {max }}$ apresentou o mesmo resultado da estatística $\lambda_{\text {traço }}$. 
Tabela 4 - Resultados do teste de co-integração, de Johansen, para a estatística $\lambda_{\text {traço }}$, variáveis $L b g$ e $L b m$, janeiro de 1995 a janeiro de 2007

\begin{tabular}{ccccccc}
\hline $\mathbf{H}_{\mathbf{0}}$ & $\mathbf{H}_{\mathbf{1}}$ & Eigenvalue & $\lambda_{\text {traço }}$ & $\begin{array}{c}\text { Valor } \\
\text { crítico }\end{array}$ & $\begin{array}{c}\text { Intercepto no } \\
\text { modelo de } \\
\text { correção de erro }\end{array}$ & $\begin{array}{c}\text { Intercepto } \\
\text { no Processo }\end{array}$ \\
\hline \hline Rank $=\mathrm{r}$ & Rank > $\mathrm{r}$ & & & & & \\
\cline { 1 - 5 } & 0 & 0,0313 & 6,81 & 12,21 & Sem Intercepto & Constante \\
1 & 1 & 0,0157 & 2,26 & 4,14 & & \\
\hline
\end{tabular}

Fonte: Dados Básicos do Instituto de Economia Agrícola (1995-2007).

Os resultados obtidos mostraram que as duas variáveis, no período considerado, não são co-integradas, isto é, não possuem relação de equilíbrio no longo prazo. Esse resultado é explicado por particularidades da cadeia produtiva, notadamente pelo ciclo pecuário plurianual, conforme Toledo e Santiago (1984).

Tendo em vista que o número de vetores é igual a zero, o modelo a ser ajustado deve ser um modelo de Auto-Regressão Vetorial (VAR), nas diferenças.

Nas Tabelas 5 e 6 são apresentados os resultados da decomposição da variância dos erros de previsão dos preços do boi gordo e do boi magro.

Tabela 5 - Resultados da proporção da decomposição da variância dos erros de previsão para a variável $L b g$

\begin{tabular}{cccc}
\hline Variável & Período a frente & $\boldsymbol{L b g}$ & $\boldsymbol{L b m}$ \\
\hline \hline \multirow{3}{*}{$\operatorname{Lbg}$} & Lead & $(\%)$ & $(\%)$ \\
\cline { 2 - 4 } & 1 & 100,00 & 0,00 \\
& 12 & 99,55 & 0,45 \\
\hline
\end{tabular}

Fonte: Dados Básicos do Instituto de Economia Agrícola (1995-2007). 
Tabela 6 - Resultados da proporção da decomposição da variância dos erros de previsão para a variável $\mathrm{Lbm}$

\begin{tabular}{cccc}
\hline Variável & Período a frente & $\boldsymbol{L b g}$ & $\boldsymbol{L b m}$ \\
\hline \hline \multirow{3}{*}{$\operatorname{lbm}$} & Lead & $(\%)$ & $(\%)$ \\
\cline { 2 - 4 } & 1 & 52,25 & 47,75 \\
& 12 & 56,38 & 43,62 \\
\hline
\end{tabular}

Fonte: Dados Básicos do Instituto de Economia Agrícola (1995-2007).

Em relação à variável $L b g$, os resultados da decomposição da variância dos erros de previsão mostraram que, em função de um choque não antecipado sobre essa variável, a maior parcela da decomposição do erro de previsão deve-se a ela própria. Mais precisamente, decorridos doze meses após a incidência de um choque não antecipado sobre os preços do boi gordo, 99,55\% da decomposição do seu erro de previsão deve-se a ela mesma, e apenas $0,45 \%$, ao comportamento dos preços do boi magro (Tabela 5), ou seja, a variável preço do boi gordo é exógena em relação à variável preço do boi magro.

Já na variável $\mathrm{Lbm}$ verifica-se um comportamento distinto, pois, decorridos doze meses após um choque não antecipado sobre ela própria, sua decomposição da variância dos erros de previsão cai de 47,75\%, após um mês, para 43,62\%, após doze meses, enquanto 56,38\% restante do comportamento dos preços do boi magro deve-se aos preços do boi gordo (Tabela 6).

Além da decomposição da variância, verificaram-se também as elasticidades das funções de resposta de impulso das variáveis estudadas, conforme mostram as Figuras 1 e 2. 


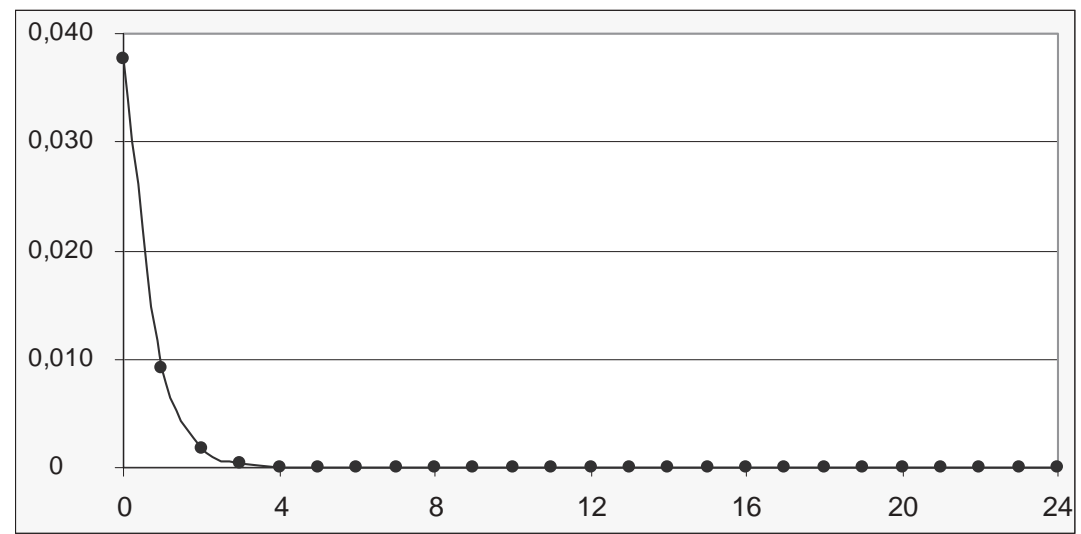

Figura 1 - Função de resposta de impulso ortogonolizada dos preços do boi magro no estado de São Paulo, em relação a variações de preços do boi gordo no estado de São Paulo, em 24 meses.

Fonte: Dados Básicos do Instituto de Economia Agrícola (1995-2007).

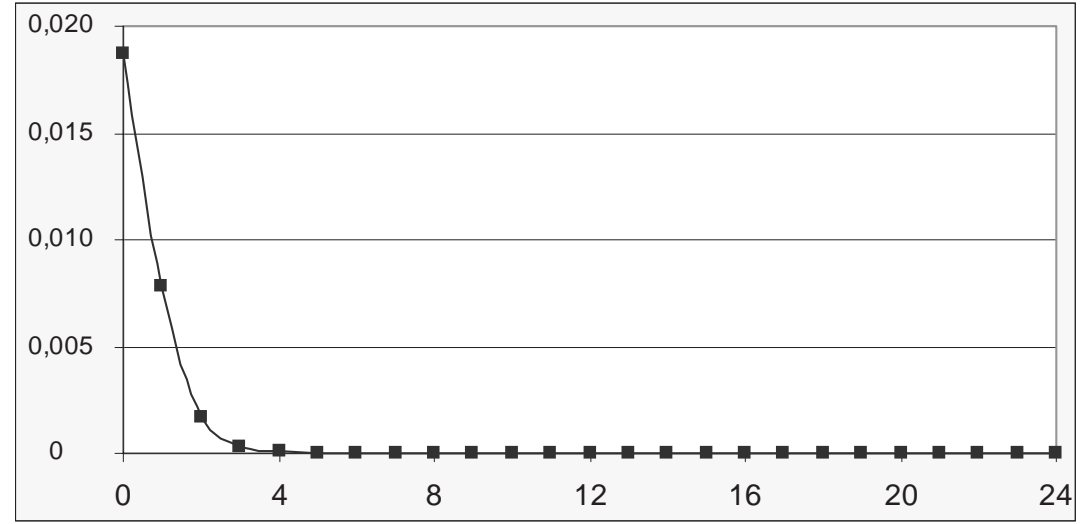

Figura 2 - Função de resposta de impulso ortogonolizada dos preços do boi gordo no estado de São Paulo, em relação a variações de preços do boi gordo no estado de São Paulo, em 24 meses.

Fonte: Dados Básicos do Instituto de Economia Agrícola (1995-2007).

A análise da função de resposta de impulso ortogonalizada mostra que variações não antecipadas nos preços do boi gordo, no estado de São 
Paulo, são transferidas para os preços do boi magro, praticamente da mesma forma que para os próprios preços do boi gordo, apontando um forte relacionamento entre eles. Inicialmente, após um choque não antecipado nos preços do boi gordo, os preços do boi magro apresentam trajetória descendente até o terceiro mês, estabilizando-se em torno de zero a partir do quarto mês (Figura 1). No caso do próprio preço do boi gordo, após o choque inicial, essa variável tende a estabilizar-se em zero, decorridos, aproximadamente, quatro meses após o choque (Figura 2). Pode-se dizer que os resultados encontrados neste trabalho mostraram que os preços do boi gordo influenciam, fortemente, os preços do boi magro. As funções de resposta de impulso encontradas demonstraram que os efeitos dos choques em ambas as variáveis, no período considerado, são de curta duração.

Esses resultados demonstram que, por ser o preço do boi gordo formado em um mercado mais concentrado e mais organizado que o mercado das demais categorias animais, esperava-se que os movimentos de preços partissem do segmento relativo ao produto acabado.

Os resultados encontrados neste trabalho corroboram os de Hasegawa (1995) e Bacchi (1999), embora as especificações dos modelos sejam diferentes. O primeiro considerou, no modelo que explica a transmissão de preços entre os segmentos da pecuária de corte, os preços do boi gordo, do bezerro, da vaca gorda, do garrote e do boi magro, enquanto o segundo considerou os preços do boi gordo, bezerro e boi magro. Podese dizer que os resultados encontrados neste trabalho mostraram, da mesma forma que nos trabalhos citados anteriormente, que o preço do boi gordo influencia fortemente os preços do boi magro. As funções de resposta de impulso encontradas nos trabalhos citados demonstram que, após um choque inicial na variável preço do boi gordo, os preços do boi magro tendem a estabilizar-se após quatro meses, o que indica que os efeitos do choque eram, no período considerado, de curta duração. 


\section{Conclusões}

Neste trabalho, verificou-se a existência de relação unidirecional entre os preços do boi gordo e do boi magro, pois constatou-se que preço do boi gordo causa o preço do boi magro, o que indica que valores passados do boi gordo ajudam a prever, de maneira mais precisa, o comportamento futuro do boi magro.

Os resultados obtidos mostraram que as duas variáveis, no período considerado, não são co-integradas, isto é, não possuem relação de equilíbrio no longo prazo, embora, no curto prazo, o preço do boi gordo influencie os preços do boi magro, conforme visto nos resultados da decomposição dos erros da variância, o que confirma a hipótese deste trabalho, de que o boi gordo influencia, sistematicamente, os demais mercados.

O preço do boi gordo explica grande parte do preço do boi magro, influência próxima dos valores encontrado por Hasegawa (1995) e Bacchi (1999).

Os efeitos dos choques nos preços dos dois segmentos da bovinocultura de corte paulista são semelhantes, o que indica expressivo relacionamento entre eles. Dada a rapidez de ajustamento de preços após um choque inicial, pode-se considerar essa cadeia eficiente. 


\section{Referências}

ANUÁRIO DE INFORMAÇÕES ESTATÍSTICA DA AGRICULTURA, 1995-2005. São Paulo, Instituto de Economia Agrícola, 1996-2006.

BACCHI, M.R.P. Previsão de preços de bovino, suíno e frango com modelos de séries temporais. Piracicaba, 1994. 173 p. Tese (Doutorado em Agronomia) - Escola Superior de Agricultura "Luiz de Queiroz", Universidade de São Paulo.

Transmissão de preços entre os segmentos produtivos da pecuária de corte brasileira. In: CONGRESSO BRASILEIRO DE ECONOMIA E SOCIOLOGIA RURAL, 37., 1999, Foz do Iguaçu, PR. Anais eletrônicos... Brasília: SOBER, 1999. 1CD.

BLISKA, F.M de M. Transmissão de preços de carne bovina entre níveis de mercado: uma aplicação do modelo de auto-regressão vetorial. Piracicaba, 1989. 209p. Dissertação (Mestrado em Agronomia) - Escola Superior de Agricultura "Luiz de Queiroz", Universidade de São Paulo.

DICKEY, D.A.; FULLER, W.A. Likelihood ratio statistics for autoregressive time series with a unit root. Econometrica, v.49, n.4, p.1057-1072, jul.,1981.

Distribution of the estimators for autoregressive time series with a unit root. Journal of The American Statistical Association, v.74, n.366, p.427-431, jun.,1979.

GUJARATI, D.N. Basic econometrics. 3.ed. New York: McGrawHill, 1995. 838p.

HARRIS, R.I.D. Cointegration analysis in econometric modeling. London: Prentice Hall, 1995.176p. 
HASEGAWA, M.M. O Mercado de reposição da pecuária bovina de corte no Estado de São Paulo. Piracicaba, 1995. 142 p. Dissertação (Mestrado em Agronomia) - Escola Superior de Agricultura "Luiz de Queiroz", Universidade de São Paulo.

INFORMAÇÕES ECONÔMICAS, 2005. São Paulo, Instituto de Economia Agrícola, v.36, n.1-2, jan-fev, 2007.

JOHANSEN, S.; JUSELIUS, K. Maximum Likelihood estimation and inference on cointegration with applications to the demand for money. Oxford Bulletin of Economics and Statistics, v. 52,n. 2, p. 169-210, 1990.

KASSOUF, A.L. Previsão de preços na pecuária de corte do Estado de São Paulo. Piracicaba, 1988. 102 p. Dissertação (Mestrado em Agronomia) - Escola Superior de Agricultura "Luiz de Queiroz", Universidade de São Paulo.

MACKINNON, J.G. Critical Values for Cointegration tests. In: Engle, R.F.; Granger, W.J. Long-Run economic relantionships: readings in cointegration. New York: Oxford University Press, 1991. p. 267276.

MARGARIDO, M. A. Análise da Formação de Preços no Mercado Internacional de Soja: o caso do Brasil. Agricultura em São Paulo, São Paulo, v. 47, n. 2, jul.-dez.2002.

PINO, F.A.;ROCHA, M.B. Transmissão de preços de soja no Brasil. Revista de Economia e Sociologia Rural, Brasília, v.32. n.4 (out/ dez), p.345-361, 1994

TOLEDO, Y.I.M.; SANTIAGO, M.M.D. Análise do comportamento de preços na pecuária bovina, Estado de São Paulo, 1970-83. Informações Econômicas, São Paulo, v. 14, n. 6, jun.1984, out. dez., 1994. 
Abstract - The paper analyzed the impacts of the price of finishing steers on the price of backgrounding steers in the State of São Paulo from January 1995 to January 2007. The ADF Unit Root test, the Granger causality test, the Johansen's co-integration test, and the method of Vector Auto-Regression (VAR) were used. In this period, the results indicated that exist a unidirectional relationship among the prices of finishing steers and backgrounding steers, confirming the importance of the prices of finishing steers on the prices formation of the backgrounding steers, but the prices had no equilibrium relationship in the long term.

Key-words: finishing steer, backgrounding steers, co-integration, vector auto-regression model (VAR), beef cattle. 
REVISTA DE ECONOMIA E AGRONEGÓCIO, VOL.5, $N^{o} 3$ 